\title{
Polymorphisms of the endothelial nitric oxide synthase gene in breast cancer: a genetic association study and meta-analysis
}

Elias Zintzaras, Maria Grammatikou, Georgios D Kitsios, Chrysa Doxani, Nikos Zdoukopoulos and Christos Papandreou

Journal of Human Genetics (2010) 55, 777; doi:10.1038/jhg.2010.136

Correction to: Journal of Human Genetics (2010) 55, 743-748; doi:10.1038/jhg.2010.100; published online 19 August 2010

The authors of the above paper noticed an error in publication (AOP and in November issue) in the list of authors. Anna Patrikidou should have been included among the authors. Dr Patrikidou contributed significantly to this article by playing a supervisory role during the execution of all relevant experiments. 